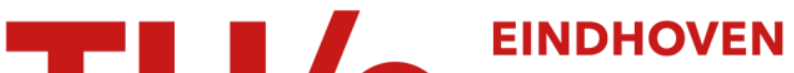 UNIVERSITY OF TECHNOLOGY
}

\section{Simulator of a Full Fetal Electrocardiogram Measurement Chain by Multichannel Capacitive Sensing}

\section{Citation for published version (APA):}

Xu, L., Rabotti, C., Zhang, Y., Vullings, R., Meftah, M., Ouzounov, S., Harpe, P. J. A., \& Mischi, M. (2020). Simulator of a Full Fetal Electrocardiogram Measurement Chain by Multichannel Capacitive Sensing. IEEE Transactions on Instrumentation and Measurement, 69(7), 4348-4357. [8873661]. https://doi.org/10.1109/TIM.2019.2947979

\section{Document license:}

TAVERNE

DOI:

10.1109/TIM.2019.2947979

Document status and date:

Published: 01/07/2020

\section{Document Version:}

Publisher's PDF, also known as Version of Record (includes final page, issue and volume numbers)

\section{Please check the document version of this publication:}

- A submitted manuscript is the version of the article upon submission and before peer-review. There can be important differences between the submitted version and the official published version of record. People interested in the research are advised to contact the author for the final version of the publication, or visit the $\mathrm{DOI}$ to the publisher's website.

- The final author version and the galley proof are versions of the publication after peer review.

- The final published version features the final layout of the paper including the volume, issue and page numbers.

Link to publication

\section{General rights}

Copyright and moral rights for the publications made accessible in the public portal are retained by the authors and/or other copyright owners and it is a condition of accessing publications that users recognise and abide by the legal requirements associated with these rights.

- Users may download and print one copy of any publication from the public portal for the purpose of private study or research.

- You may not further distribute the material or use it for any profit-making activity or commercial gain

- You may freely distribute the URL identifying the publication in the public portal.

If the publication is distributed under the terms of Article 25fa of the Dutch Copyright Act, indicated by the "Taverne" license above, please follow below link for the End User Agreement:

www.tue.nl/taverne

Take down policy

If you believe that this document breaches copyright please contact us at:

openaccess@tue.nl

providing details and we will investigate your claim. 


\title{
Simulator of a Full Fetal Electrocardiogram Measurement Chain by Multichannel Capacitive Sensing
}

\author{
Lin $\mathrm{Xu}^{{ }^{\circledR}}$, Member, IEEE, Chiara Rabotti ${ }^{\circledR}$, Member, IEEE, Yijing Zhang ${ }^{(}$, Rik Vullings ${ }^{\circledR}$, Member, IEEE, \\ Mohammed Meftah ${ }^{\circledR}$, Sotir Ouzounov ${ }^{\circledR}$, Pieter J. A. Harpe ${ }^{\circledR}$, Senior Member, IEEE, \\ and Massimo Mischi ${ }^{\circledR}$, Senior Member, IEEE
}

\begin{abstract}
L Long-term ambulatory monitoring of the fetal heart rate (fHR) can greatly increase insight into fetal well-being and reduce pregnancy risks. Unfortunately, the existing solutions using wet or dry electrodes are unsuitable for long-term fHR monitoring due to the use of a gel or direct skin contact. Capacitive electrodes can measure an electrocardiographic (ECG) signal through clothes and, therefore, are perfectly suitable for long-term monitoring of the fHR. However, capacitive fetal ECG (fECG) measurements are challenging due to the high sensitivity of capacitive sensors to motion artifacts (MAs) and the low amplitude of the fECG. This article aims at investigating the feasibility of fECG measurements using capacitive electrodes with dedicated postprocessing algorithms for signalto-noise ratio (SNR) improvement and MA reduction. To this end, a novel simulator of the full measurement chain is realized that generates multichannel capacitive fECG data with artificial MAs and system noise. A dedicated blind source separation (BSS) algorithm is then employed for MA removal and fECG extraction. The extracted fECG is evaluated by SNR calculation and R-peak detection. Our results show that the BSS algorithm may extract the fECG signal from noisy capacitive data. In addition, lower system noise or higher number of channels may lead to better fECG extraction. A maximum increase of $0.5 \mathrm{~dB}$ in the SNR and decrease of $80.7 \%$ in the R-peak detection error are observed with increased electrode number from 8 to 20. Our findings provide useful insights for the hardware design of a capacitive fECG measurement system.
\end{abstract}

Index Terms-Ambulatory monitoring, blind source separation (BSS), capacitive electrode, fetal electrocardiography, motion artifacts (MAs), simulation, unobtrusive.

Manuscript received August 25, 2019; accepted September 30, 2019 Date of publication October 17, 2019; date of current version June 9, 2020. This work was supported by a grant from the Dutch Technology Foundation STW, which is entitled Applied and Engineering Sciences in the new NWO Domain after January 1, 2017. The Associate Editor coordinating the review process was Vedran Bilas. (Corresponding author: Lin $\mathrm{Xu}$.)

L. Xu is with the School of Information Science, ShanghaiTech University, Shanghai 201210, China (e-mail: xulin1@ shanghaitech.edu.cn).

C. Rabotti and M. Meftah are with the Family Care Solutions Group, Philips Research Europe, 5656 AE Eindhoven, The Netherlands.

Y. Zhang and P. J. A. Harpe are with the Integrated Circuits Group, Department of Electrical Engineering, Eindhoven University of Technology, 5612 AP Eindhoven, The Netherlands.

R. Vullings and M. Mischi are with the Signal Processing System Group, Department of Electrical Engineering, Eindhoven University of Technology, 5612 AP Eindhoven, The Netherlands.

S. Ouzounov is with the Micro Systems and Devices Group, Philips Research Europe, 5656 AE Eindhoven, The Netherlands.

Color versions of one or more of the figures in this article are available online at http://ieeexplore.ieee.org.

Digital Object Identifier 10.1109/TIM.2019.2947979

\section{INTRODUCTION}

D ESPITE the rapid development in healthcare technology, pregnancies still suffer from complications, such as fetal growth restriction, preterm birth, or stillbirth [1]. Although the origins of these pregnancy-related complications are still poorly understood, the fetal heart rate (fHR) is among the most important features used to assess the fetal well-being and the progression of pregnancy. Doppler ultrasound is currently clinically used to measure the fHR by placing a transducer on the maternal abdomen, as part of a cardiotocograph [2]. The fetal electrocardiogram (fECG), measured invasively during labor by applying an electrode to the fetal scalp or noninvasively by placing wet electrodes on the maternal abdomen, has also been used to monitor the fHR. One of the main drawbacks of these techniques is that none of these systems is suitable for long-term ambulatory monitoring of fHR.

Capacitive electrodes have been introduced in ambulatory ECG monitoring for adults a couple of decades ago and further developed in recent years [3], [4]. Capacitive electrodes enable ECG measurements through clothes, with signal quality comparable to that measured by contact wet electrodes [5]. Furthermore, it is easy to integrate capacitive electrodes into daily objects, such as a bed, a chair, a car seat, and even a neonatal incubator [6]-[9]. The main challenge in a capacitive ECG measurement system is the high sensitivity to motion artifacts (MAs) [10]-[12].

MAs originate mainly from longitudinal and lateral movements of the electrodes with respect to the body [10]. The longitudinal movement produces variation in the coupling distance and, therefore, changes the coupling capacitance. Change in the coupling capacitance can modulate the desired ECG signal and the voltage difference (dc) between the body and the measurement system, introducing both multiplicative and additive MAs [13]. The lateral movement causes friction at the body-electrode interface, and thus, introduces triboelectricity [11], [12]. The amplitude of triboelectricity can be as high as several volts, and its occurrence is unpredictable [12]. As a result, MAs may mask the signal of interest and, therefore, should be addressed prior to adopting a capacitive ECG measurement system for ambulatory monitoring.

The presence of these MAs makes capacitive ECG measurements very challenging for adults; the significantly smaller amplitude of the fECG as compared with adults further 

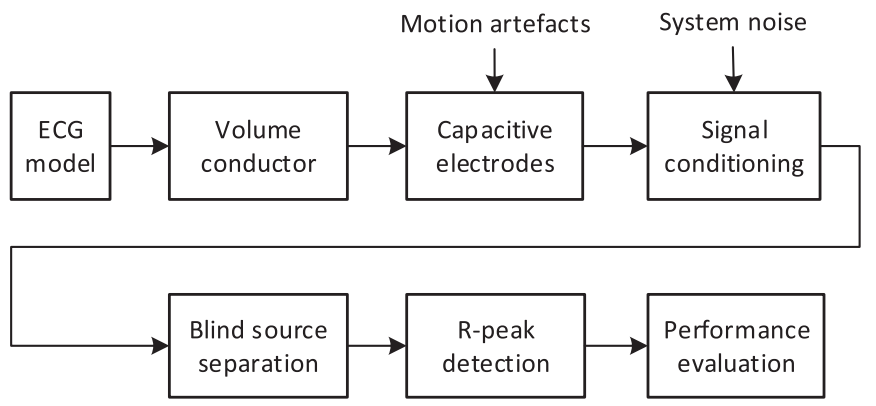

Fig. 1. Scheme of the simulator of the full capacitive measurement chain.

increases the complexity of capacitive fECG measurements. As a consequence, no existing capacitive system has been reported to be able to measure a fECG. The aim of this article is, therefore, to investigate the feasibility of using capacitive electrodes for fECG measurements by dedicated in - silico simulation. To this end, a multichannel simulator of a full capacitive fECG measurement chain is realized for the first time by integrating existing models that have been individually investigated by previous authors, such as the ECG model and the model of the capacitive sensors [11], [14]-[17]. The realized simulator generates multichannel capacitive fECG data with artificial MAs and system noise. Varying electrode number and system noise are separately simulated, enabling the examination of the influence of system noise and number of channels on the performance of the full capacitive fECG measurement chain, and thus providing useful insights on the hardware design of a capacitive fECG measurement system.

Dedicated algorithms are embedded in the simulator in order to reduce the MAs, improve the signal-to-noise ratio (SNR), and therefore, extract the fECG. Previous authors have suggested several methods for MA reduction in capacitive adult ECG measurements, such as the reconstruction of the ECG signal by solving the inverse system function, estimation of model parameters by signal injection, adaptive filtering, and blind source separation (BSS) based on multichannel recordings [8], [11], [13], [18], [19]. Among all the available methods, BSS seems to produce robust ECG extraction in adults [7], [8], [19] and is, therefore, adopted in our simulator for MA reduction and FECG extraction. The performance of the fECG extraction is quantitatively evaluated by calculating the SNR and detecting the R-peaks on the extracted fECG signal.

\section{METHOD}

\section{A. Description of the Simulator}

The simulator of the full capacitive fECG measurement chain is realized by integrating existing individual models [11], [14], [16], [17], whose scheme is shown in Fig. 1. First, an ECG model is employed to generate the maternal and fetal cardiac electrical activity, which is known as vectorcardiogram (VCG). The generated VCG waveforms are then projected onto the surface of the volume conductor. The potentials on the surface of the volume conductor are detected by capacitive electrodes and then amplified and digitized by the following signal conditioning hardware. The aforementioned two types of MAs are introduced in the capacitive electrode model, and white Gaussian noise is added to mimic the system noise, e.g., thermal noise. A BSS algorithm is applied to the simulated signals in order to extract the fECG. R-peaks of the extracted fECG signal are then detected and evaluated.

1) ECG Model: The ECG model described in [20] is adopted in this article in order to generate the maternal and fetal VCG waveforms, which can be represented by a time-varying dipole vector with its origin at the center of the heart. In general, the heart dipole vector can be expressed as

$$
\underline{\mathbf{d}}(t)=x(t) \underline{\mathbf{e}}_{x}+y(t) \underline{\mathbf{e}}_{y}+z(t) \underline{\mathbf{e}}_{z}
$$

where $\underline{\mathbf{e}}_{j}, j \in\{x, y, z\}$, are basis vectors of the body axis. According to the dynamic ECG model described in [20], the projection of one VCG cycle along each body axis can be approximated by the summation of a set of Gaussian kernel functions as

$$
\mathrm{ECG}_{j}(\boldsymbol{\theta})=\sum_{i=1}^{N} a_{j i} e^{-\frac{\left(\theta-\mu_{j i}\right)^{2}}{2 \sigma j i i^{2}}}, \quad-\pi \leq \theta \leq \pi
$$

where $N$ is the number of the Gaussian kernel functions and $a_{j i}, \mu_{j i}$, and $\sigma_{j i}$ correspond to the peak amplitude, peak location, and standard deviation of the $i$ th Gaussian kernel function, respectively. By fitting this model to a set of existing VCGs, i.e., all three channels of the VCGs, from an existing database, the model parameters on each axis $j, a_{j i}, \mu_{j i}$, and $\sigma_{j i}$, can be obtained.

After obtaining $a_{j i}, \mu_{j i}$, and $\sigma_{j i}$, the first derivatives of the three coordinates of the time-varying heart dipole, $\underline{\mathbf{d}}(t)$, can then be calculated as [14], [20]

$$
\begin{aligned}
\dot{\boldsymbol{\theta}} & =2 \pi f_{h} \\
\dot{x} & =-\sum_{i=1}^{N} \frac{a_{x i} \dot{\boldsymbol{\theta}}}{\left(\sigma_{x i}\right)^{2}} \Delta \boldsymbol{\theta}_{x i} \exp \left[-\frac{\left(\Delta \boldsymbol{\theta}_{x i}\right)^{2}}{2\left(\sigma_{x i}\right)^{2}}\right] \\
\dot{y} & =-\sum_{i=1}^{N} \frac{a_{y i} \dot{\boldsymbol{\theta}}}{\left(\sigma_{y i}\right)^{2}} \Delta \boldsymbol{\theta}_{y i} \exp \left[-\frac{\left(\Delta \boldsymbol{\theta}_{y i}\right)^{2}}{2\left(\sigma_{y i}\right)^{2}}\right] \\
\dot{z} & =-\sum_{i=1}^{N} \frac{a_{z i} \dot{\boldsymbol{\theta}}}{\left(\sigma_{z i}\right)^{2}} \Delta \boldsymbol{\theta}_{z i} \exp \left[-\frac{\left(\Delta \boldsymbol{\theta}_{z i}\right)^{2}}{2\left(\sigma_{z i}\right)^{2}}\right]
\end{aligned}
$$

with

$$
\Delta \boldsymbol{\theta}_{j i}=\left(\boldsymbol{\theta}-\mu_{j i}\right) \bmod 2 \pi, \quad j \in\{x, y, z\}
$$

where $f_{h}$ is the beat-to-beat HR.

Furthermore, the normal sinus rhythm (NSR) modulation described in [20] can be employed to introduce HR variability (HRV) in this model, permitting to produce maternal and fetal signals in both physiological and pathological scenarios [14]. According to [20], the effect of the NSR modulation on the spectrum of the RR-intervals can be modeled as the sum of two Gaussian distributions

$$
R R(f)=\frac{A_{1}}{\sqrt{2 \pi c_{1}^{2}}} \exp \left[\frac{\left(f-f_{1}\right)^{2}}{2 c_{1}^{2}}\right]+\frac{A_{2}}{\sqrt{2 \pi c_{2}^{2}}} \exp \left[\frac{\left(f-f_{2}\right)^{2}}{2 c_{2}^{2}}\right]
$$

with means $f_{1}$ and $f_{2}$, amplitudes $A_{1}$ and $A_{2}$, and standard deviations $c_{1}$ and $c_{2}$. The beat-to-beat HR $f_{h}$ in (6) is then 
replaced by a time-dependent HR as given by

$$
f(t)=f_{h}+\frac{1}{\operatorname{ifft}\{R R(f)\}}
$$

where ifft denotes the inverse Fourier transform.

2) Volume Conductor: The generated maternal and fetal VCG waveforms are projected on the surface of a volume conductor by a projection matrix $\mathbf{H}(t)$ and a rotation matrix $\mathbf{R}(t)$, as given by

$$
\underline{\mathbf{p}}(t)=\mathbf{H}(t) \times \mathbf{R}(t) \times\left[\begin{array}{l}
x(t) \\
y(t) \\
z(t)
\end{array}\right]
$$

where $\mathbf{p}(t)$ is the potential detected at the electrode position on the volume conductor, $\left[\begin{array}{lll}x(t) & y(t) & z(t)\end{array}\right]^{T}$ contains the three coordinates of the dipole vector generated by the model described in (6), $\mathbf{H}(t)$ is the projection matrix representing the volume conductor which is time varying in cases where the dipole is translated [14], and $\mathbf{R}(t)$ is the rotation matrix of the dipole vector.

The projection matrix $\mathbf{H}(t)$ is determined by the permittivity of the volume conductor, the dipole origin, and the vector going from the dipole center to the location of the detection electrode, denoted as $\underline{\mathbf{r}}(t)$. By assuming a constant permittivity, $\varepsilon_{0}$, the projection matrix $\mathbf{H}(t)$ can be rewritten as $\mathbf{H}(t)=$ $\left[\begin{array}{lll}h_{x}(t) & h_{y}(t) & h_{z}(t)\end{array}\right]$, with $h_{j}(t)$ given as

$$
h_{j}(t)=\frac{1}{4 \pi \varepsilon_{0}} \frac{r_{j}(t)}{|\underline{\mathbf{r}}(t)|^{3}}, \quad j \in\{x, y, z\} .
$$

Since respiration can influence the orientation of the cardiac dipole and, therefore, the rotation matrix $\mathbf{R}(t)$, the respiration model introduced by Stridh and Sörnmo [21] is considered in the derivation of the rotation matrix $\mathbf{R}(t)$. In this model, a sinusoid and two harmonics are employed to generate a sawtooth-like signal $\gamma(t)$ representing the respiration pattern. Nonstationary behavior of the physiological process is introduced by modulating both the amplitude and frequency of each sinusoid, as given by

$$
\begin{aligned}
& \gamma(t)=-\sum_{k=1}^{3} \alpha_{k}(t) \cdot \sin \left[k \cdot 2 \pi f_{0} t+\frac{\Delta f}{f_{m}} \sin \left(2 \pi f_{m} t\right)\right] \\
& \alpha_{k}(t)=\frac{2}{k \pi}\left(\alpha+\Delta \alpha \cdot \sin \left(2 \pi f_{\alpha} t\right)\right)
\end{aligned}
$$

with $\alpha$ the amplitude of each sinusoid and $f_{0}$ the fundamental frequency corresponding to the breathing rate. $f_{m}$ and $\left(\Delta f / f_{m}\right)$ represent the frequency and strength for the frequency modulation, respectively, while $f_{\alpha}$ and $\Delta \alpha$ the frequency and strength for the amplitude modulation, respectively.

By taking the respiration model into consideration, the rotation matrix $\mathbf{R}(t)$ can be written as

$$
\mathbf{R}(t)=\mathbf{R}_{x}(t) \cdot \mathbf{R}_{y}(t) \cdot \mathbf{R}_{z}(t)
$$

where

$$
\begin{aligned}
\mathbf{R}_{x}(t) & =\left[\begin{array}{ccc}
1 & 0 & 0 \\
0 & \cos \left[\varphi_{x}(t)\right] & \sin \left[\varphi_{x}(t)\right] \\
0 & -\sin \left[\varphi_{x}(t)\right] & \cos \left[\varphi_{x}(t)\right]
\end{array}\right] \\
\mathbf{R}_{y}(t) & =\left[\begin{array}{ccc}
\cos \left[\varphi_{y}(t)\right] & 0 & \sin \left[\varphi_{y}(t)\right] \\
0 & 1 & 0 \\
-\sin \left[\varphi_{y}(t)\right] & 0 & \cos \left[\varphi_{y}(t)\right]
\end{array}\right] \\
\mathbf{R}_{z}(t) & =\left[\begin{array}{ccc}
\cos \left[\varphi_{z}(t)\right] & \sin \left[\varphi_{z}(t)\right] & 0 \\
-\sin \left[\varphi_{z}(t)\right] & \cos \left[\varphi_{z}(t)\right] & 0 \\
0 & 0 & 1
\end{array}\right]
\end{aligned}
$$

with

$$
\varphi_{j}(t)=\varphi_{j}^{\max }(t) \cdot \gamma(t)+\varphi_{j}^{0}, \quad j \in\{x, y, z\}
$$

where $\varphi_{j}(t)$ is the rotation angle in radians around the axis $j$, $\varphi_{j}^{0}$ is the the static orientation of the fetal heart with respect to the maternal heart, which is assumed to be aligned with the Cartesian basis, and $\varphi_{j}^{\max }(t)$ is the maximum deviation of the rotation angle $\varphi_{j}(t)$.

3) Capacitive System: The potential projected onto the surface of the volume conductor is then detected by capacitive electrodes. A capacitive electrode is typically composed of a plate electrode followed by a buffer with very high-input impedance for lower cutoff frequencies and a lower noise, as shown in Fig. 2(a) [4]. Active guarding is applied to prevent environmental electric field from coupling into the measurement system, reducing the power-line interference in the measured signal. A bias resistor is adapted to create a current path to the ground [7]. In addition, an isolation layer is placed on top of the electrode plate in order to create a capacitive system. The coupling layer between body and electrode can be air or cloth.

The isolation layer can be modeled as a capacitor in parallel with a resistor [12]. The capacitance is in the order of $\mathrm{pF}$, e.g., $20 \mathrm{pF}$, and the resistance in the order of $10^{14} \Omega$ [12]. The impedance of the capacitor depends on the frequency of the signal. Consider a capacitance of $20 \mathrm{pF}$ [12] and a very low frequency, e.g., $0.5 \mathrm{~Hz}$, the impedance of the capacitor is approximately $1.59 \times 10^{10} \Omega$, which is much smaller than the impedance of the resistor and is, therefore, dominant in the insolation layer. Consequently, some studies simply model the isolation layer as a pure capacitor and neglect this large resistor [10]. This simplified model is adopted in this article.

The coupling layer, air or cloth, can also be modeled as a capacitor in parallel with a resistor [10], [12]. The capacitance $C_{c}(t)$ is calculated as

$$
C_{c}(t)=\frac{\varepsilon_{r} A}{d(t)}
$$

where $\varepsilon_{r}$ is the dielectric constant of the medium, $A$ is the area of the electrode surface, and $d(t)$ is the distance between body and electrode. $C_{c}(t)$ is considered to be time varying due to movement-induced variation in $d(t)$.

Taking the triboelectric effect into consideration, the capacitive system shown in Fig. 2(a) can be represented by an equivalent circuit as shown in Fig. 2(b), where $v_{\text {fecg }}(t)$ and $v_{\text {mecg }}(t)$ represent the fECG and maternal ECG (mECG), respectively. $R_{i}$ and $C_{i}$ represent the input impedance of the buffer. $R_{B}$ is 


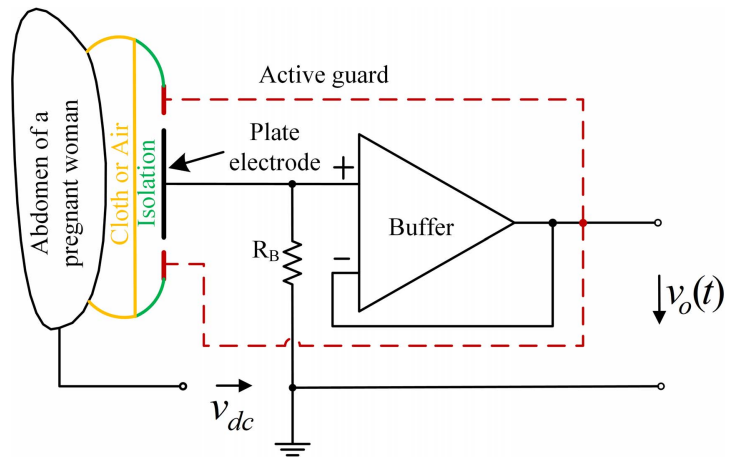

(a)

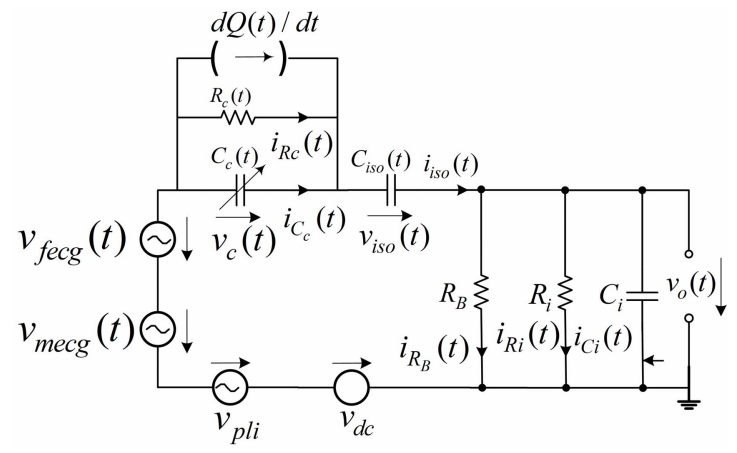

(b)

Fig. 2. Capacitive ECG measurement system: (a) physical model and (b) equivalent circuit including the triboelectric effect.

the bias resistor. $v_{\text {pli }}$ models the power-line interference due to displacement current coupled into the body, and $v_{\mathrm{dc}}$ accounts for the dc voltage difference between the body surface and the measurement system ground. A charge transfer between the body-electrode interface, $d Q(t) / d t$, is employed to model the effect of triboelectricity, as suggested in [12].

Kirchhoff's second law leads to

$$
v_{c}(t)=v_{\mathrm{fecg}}(t)+v_{\text {mecg }}(t)+v_{\mathrm{pli}}+v_{\mathrm{dc}}-v_{o}(t)-v_{\text {iso }}(t) .
$$

And Kirchhoff's first law results in

$$
\begin{aligned}
i_{C_{c}}(t)+i_{R_{c}}(t)+\frac{d Q(t)}{d t} & =i_{C_{\text {iso }}}(t) \\
i_{C_{\text {iso }}}(t) & =i_{C_{i}}(t)+i_{R_{i}}(t)+i_{R_{B}}(t) .
\end{aligned}
$$

Then, we obtain

$$
i_{C_{c}}(t)+i_{R_{c}}(t)+\frac{d Q(t)}{d t}=i_{C_{i}}(t)+i_{R_{i}}(t)+i_{R_{B}}(t) .
$$

The equivalent circuit shown in Fig. 2(b) can then be described by a differential equation describing the balance of electrical currents as

$$
\frac{d\left[C_{c}(t) v_{c}(t)\right]}{d t}+\frac{v_{c}(t)}{R_{c}}+\frac{d Q(t)}{d t}=\frac{d\left[C_{i} v_{o}(t)\right]}{d t}+\frac{v_{o}(t)}{R_{\mathrm{comb}}}
$$

where

$$
R_{\mathrm{comb}}=R_{i} / / R_{B} \approx R_{B}, \quad\left(R_{i} \gg R_{B}\right) .
$$

From (22), we have

$$
\frac{d\left[C_{\mathrm{iso}}(t) v_{\mathrm{iso}}(t)\right]}{d t}=\frac{d\left[C_{i} v_{o}(t)\right]}{d t}+\frac{v_{o}(t)}{R_{\mathrm{comb}}} .
$$

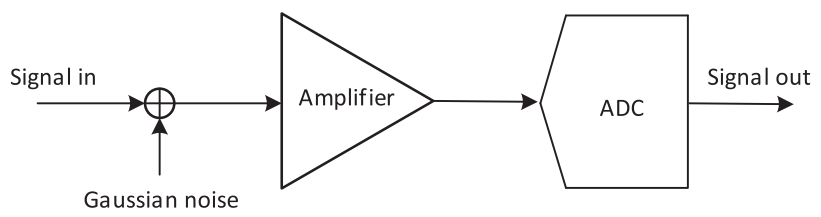

Fig. 3. Scheme of the signal conditioning hardware.

Solving (26) and assuming initial $v_{o}(t)$ to be zero, $v_{\text {iso }}(t)$ can be derived as

$$
v_{\text {iso }}(t)=\frac{C_{i}}{C_{\text {iso }}}+\frac{1}{C_{\text {iso }} R_{\text {comb }}} \int v_{o}(t) d t .
$$

Substituting $v_{\text {iso }}(t)$ into (20) with (27), we obtain

$$
\begin{aligned}
v_{c}(t)=v_{\mathrm{fecg}}(t)+v_{\mathrm{mecg}}(t)+v_{\mathrm{pli}}+v_{\mathrm{dc}} \\
-v_{o}(t)-\left[\frac{C_{i}}{C_{\mathrm{iso}}}+\frac{1}{C_{\mathrm{iso}} R_{\mathrm{comb}}} \int v_{o}(t) d t\right] .
\end{aligned}
$$

Finally, the output of the capacitive system $v_{o}(t)$ can be derived by substituting $v_{c}(t)$ into (24) with (28), which allows a numerical simulation and analysis of the capacitive system in MATLAB.

4) Signal Conditioning: The signal detected by the capacitive electrodes is amplified and digitized by the signal-conditioning hardware. Since the focus of this article is the full measurement chain of fECG using capacitive electrodes, the adopted signal-conditioning hardware in the simulator simply consists of a voltage amplifier and an analogto-digital converter (ADC), as shown in Fig. 3. White Gaussian noise is added to the simulator to model the system noise introduced by the conditioning hardware, e.g., the thermal noise. The SNR between the fECG and the added system noise is defined as

$$
\mathrm{SNR}_{\text {in }}=10 \cdot \log \frac{P_{s}}{P_{n}}
$$

where $P_{S}$ is the signal power of the fECG and $P_{n}$ the noise power.

5) Blind Source Separation: BSS approaches, particularly independent component analysis (ICA), have been used for extracting the fECG measured by wet electrodes [19] or extracting the adult ECG measured by capacitive electrodes [8]. In this article, ICA is proposed for extracting the fECG from noisy capacitive measurements. A typical way to formulate the ICA approach is to assume the observed data to be a linear combination of different independent sources, as given by [22], [23]

$$
\mathbf{x}=\mathbf{A s}
$$

where $\mathbf{x}$ is the observed n-dimensional data, $\mathbf{s}$ is an m-dimensional random vector with its components mutually independent, and $\mathbf{A}$ is the $n \times m$ mixing matrix. The objective of ICA is to estimate the mixing matrix $\mathbf{A}$, and thus, reconstruct the independent sources by

$$
\mathbf{s}=\mathbf{W} \mathbf{x}
$$

where $\mathbf{W}=\mathbf{A}^{-1}$.

Non-Gaussianity of the independent components is necessary for the identification of $\mathbf{W}$ [24], [25]. One measure of 
TABLE I

PARAMETERS FOR INTRODUCING HRV (8)

\begin{tabular}{llllll}
\hline & $f_{1}[\mathrm{~Hz}]$ & $f_{2}[\mathrm{~Hz}]$ & $c_{1}[\mathrm{~Hz}]$ & $c_{2}[\mathrm{~Hz}]$ & $A_{1} / A_{2}$ \\
\hline Fetal & 0.32 & 0.8 & 0.025 & 0.025 & 0.5 \\
Maternal & 0.1 & 0.25 & 0.015 & 0.015 & 0.5 \\
\hline
\end{tabular}

non-Gaussianity is the negentropy, defined by $J(s)$ as [24], [25]

$$
\begin{aligned}
J(\mathbf{s}) & =H\left(\mathbf{s}_{\text {Gauss }}\right)-H(\mathbf{s}) \\
H(\mathbf{s}) & =-\int p(\mathbf{s}) \log p(\mathbf{s}) d \mathbf{s}
\end{aligned}
$$

where $p(\mathbf{s})$ is the probability density of the random variable $\mathbf{s}$ and $\mathbf{s}_{\text {Gauss }}$ is a Gaussian random variable with the same covariance matrix as $\mathbf{s}$.

Based on the negentropy, one can define the mutual information, $I$, between the $m$ components $s_{i}, i=1, \ldots, m$ :

$$
I\left(s_{1}, s_{2}, \ldots, s_{m}\right)=J(\mathbf{s})-\sum_{i} J\left(s_{i}\right) .
$$

Mutual information is a natural measure of the dependence between the $m$ components [24], [25]. By minimizing the mutual information between the $m$ components $s_{i}$, the matrix W can be estimated.

\section{B. In-Silico Experiment}

1) VCG Generation: A 60-s fetal VCG and maternal VCG were first generated based on the ECG model described in (6), whose parameters $a_{j i}, \mu_{j i}$, and $\sigma_{j i}$ were derived from five existing VCGs in the PTB diagnostic ECG database records [26]. The SNR between the fECG and $\mathrm{mECG}$ was set to $-10 \mathrm{~dB}$, in line with previous studies [14]. The mean HR was 90 beats per minute (bpm) for the mother and $150 \mathrm{bpm}$ for the fetus. The parameters adopted in [14] and [20], shown in Table I, were employed for the NSR modulation in order to introduce HRV using (8).

2) Projection of VCG Waveforms: A cylinder with unit diameter and height was employed to mimic the volume conductor, as shown in Fig. 4. The maternal and fetal dipoles were represented by three arrows that show the orientation of the fetal and maternal body axis centered on each heart. Initially, the maternal dipole was assumed to be aligned with the maternal body axis while the fetal dipole was modeled to have a rotation of $-3 \pi / 4$ along the $x$-axis and $-\pi / 2$ along the $z$-axis with respect the maternal dipole. Twenty capacitive electrodes were placed on the surface of the cylinder to detect the projection of the fetal and maternal dipoles. An additional capacitive electrode (no. 21) was placed far from both hearts to detect a reference signal. The positions of the electrodes are reported in Table II together with the position of the fetal and maternal hearts.

Equation (10) was used to project the fetal and maternal dipole vectors (VCG) to the detection position of the electrodes, in which the rotation matrix $\mathbf{R}(t)$ was calculated by taking the respiration pattern $\gamma(t)$ into consideration (13). For $\gamma(t)$, the fetal and maternal breathing rate were set to 0.8 and $0.25 \mathrm{~Hz}$, respectively [14]. Other parameters were:

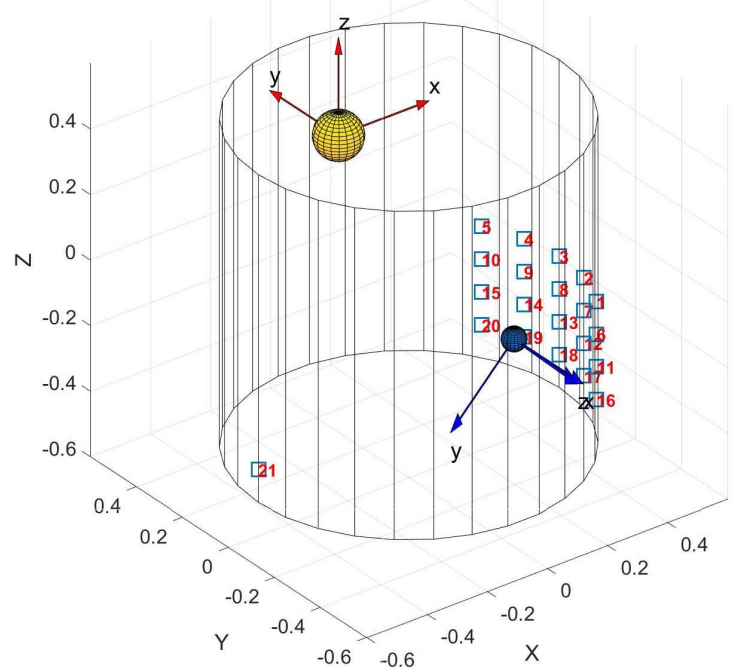

(a)

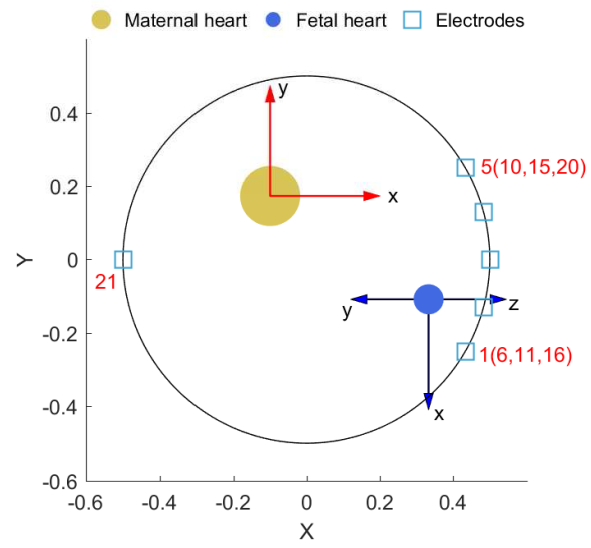

(b)

Fig. 4. Volume conductor. (a) Three-dimensional view. (b) Top view.

TABLE II

Electrodes And HeArt Positions; $\psi$ THE Angle (IN rad), $\rho$ THE RADIUS, AND $h$ THE HEIGHT

\begin{tabular}{llll}
\hline & $\psi$ & $\rho$ & $h$ \\
\hline m-heart & $2 \pi / 3$ & 0.2 & 0.4 \\
f-heart & $-\pi / 10$ & 0.35 & -0.4 \\
Electrodes $1-5$ & $-2 \pi / 12,-\pi / 12,0, \pi / 12,2 \pi / 12$ & 0.5 & -0.1 \\
Electrodes $6-10$ & $-2 \pi / 12,-\pi / 12,0, \pi / 12,2 \pi / 12$ & 0.5 & -0.2 \\
Electrodes 11-15 & $-2 \pi / 12,-\pi / 12,0, \pi / 12,2 \pi / 12$ & 0.5 & -0.3 \\
Electrodes 16-20 & $-2 \pi / 12,-\pi / 12,0, \pi / 12,2 \pi / 12$ & 0.5 & -0.4 \\
Reference & $-\pi$ & 0.5 & -0.4 \\
\hline
\end{tabular}

$\Delta \alpha=0.3, f_{\alpha}=0.1 \mathrm{~Hz}, \Delta f=0.05 \mathrm{~Hz}$, and $f_{m}=0.1 \mathrm{~Hz}$, as adopted in [14] and [20].

3) Capacitive Sensing: The differential equation, (24), describing the dynamic behavior of the capacitive electrodes was implemented in Simulink (MathWorks, Natick, MA, USA). The capacitance of the isolation layer was set to $20 \mathrm{pF}$ [12]. A cotton cloth with a resistance of $200 \mathrm{M} \Omega$ was assumed between body and electrode [10]. A circular surface with diameter of $2 \mathrm{~cm}$ was assumed for the capacitive electrode [11], and a chirp motion from 0.2 to $10 \mathrm{~Hz}$, which covers the full frequency range of body movement [27], was employed to mimic the longitudinal movement between 
TABLE III

Parameters Used to Model a Capacitive ELECTRODE INCLUDING MAS

\begin{tabular}{lll}
\hline Parameters & Symbol & Value \\
\hline Input resistance & $R_{i}$ & $1000 \mathrm{G} \Omega$ \\
Input capacitance & $C_{i}$ & $2 \mathrm{pF}$ \\
Bias resistance & $R_{B}$ & $50 \mathrm{G} \Omega$ \\
DC voltage & $v_{d c}$ & $20 \mathrm{mV}$ \\
Electrode surface & $A$ & $3.14 \mathrm{~cm}^{2}$ \\
Dielectric constant & $\varepsilon_{r}$ & 4 \\
Coupling distance & $d(t)$ & $0.7 \pm(0.1 \sim 0.25) \mathrm{mm}$ \\
Resistance of cotton & $R_{c}$ & $200 \mathrm{M} \Omega$ \\
Capacitance of isolation & $C_{i s o}$ & $20 \mathrm{pF}$ \\
Charge transfer & $d Q(t) / d t$ & $20 \sim 60 \mathrm{pA}$ \\
Power-line interference & $v_{p l i}$ & $10 \mathrm{mV}$ \\
\hline
\end{tabular}

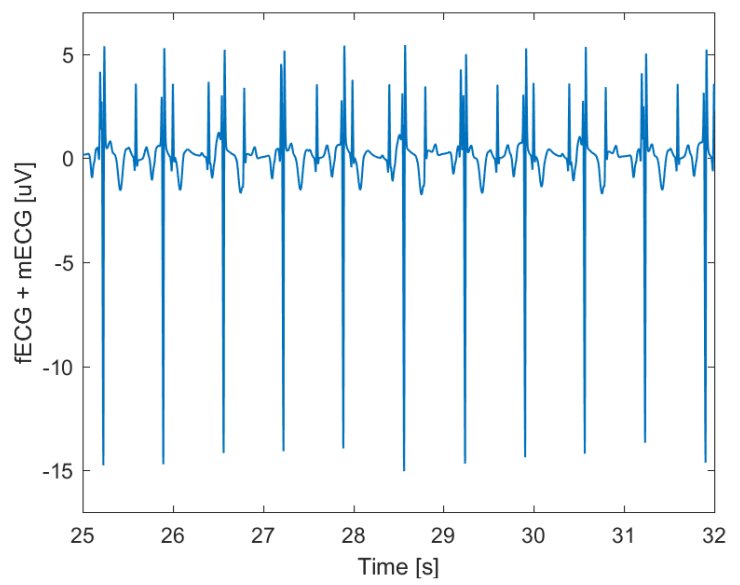

Fig. 5. Mixture of maternal and fetal ECG appearing on the surface of the cylinder that models the body.

electrode and body. For each channel, the frequency range of the chirp motion was the same, but the amplitude varied randomly. In addition, a pulse current starting at $30 \mathrm{~s}$ and lasting for $5 \mathrm{~ms}$ [12] was introduced to mimic the effect of triboelectricity. The occurrence and duration of the pulse current were the same for different channels while the amplitudes varied randomly. No MAs were added to the reference channel. The parameters adopted in the present simulator are shown in Table III. All these parameters were chosen to be either in line with literature [7], [10], [11], [13], or close to reality.

4) Signal Conditioning and Blind Source Separation: The signal conditioning circuit consisted of a voltage amplifier and an ADC and was simulated in Simulink. The gain of the amplifier was 100, and the resolution of the ADC was $20 \mathrm{~b}$. By including the $\mathrm{ADC}$, quantization noise was considered in our simulator. Furthermore, white Gaussian noise was added to each channel to mimic the system noise, e.g., the thermal noise. The fixed-point fast ICA described in [25] was applied to the output of the conditioning hardware in order to extract the fECG. Monopolar configurations were considered by taking the difference between the active electrodes (1-20) and the reference electrode.

\section{Evaluation}

For each component obtained from the fast ICA decomposition, the locations of the fetal R-peaks were detected by a low-complexity R-peak detection algorithm described in [28].
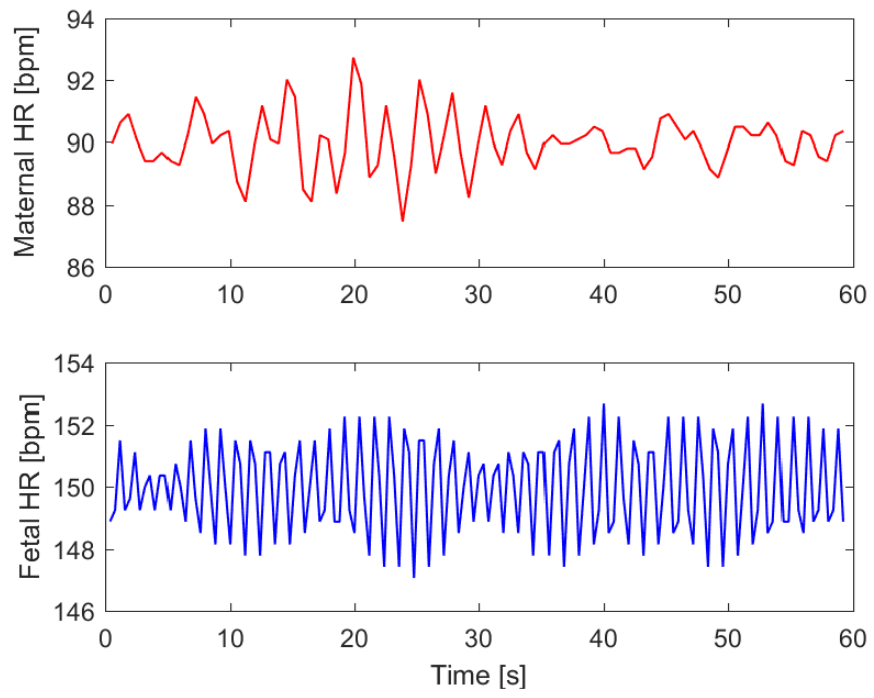

Fig. 6. Time-varying HRs of the mother and fetus.

To assess the detectability of the fECG R-peaks, a modified SNR was defined as [28]

$$
\mathrm{SNR}_{\text {out }}=\sum_{\mathrm{i}=1}^{\mathrm{P}} 20 \cdot \log \frac{v_{\mathrm{ppi}}}{v_{\mathrm{ni}}}
$$

where $P$ is the number of fECG R-peaks, $v_{\text {ppi }}$ is the maximum value of the $i$ th $\mathrm{R}$ peak, and $v_{\mathrm{ni}}$ is the maximum value in two surrounding intervals of the $i$ th R-peak. For the $i$ th R-peak, the surrounding two intervals were defined as $[t(i-1)+$ $300 \mathrm{~ms}, t(i)-75 \mathrm{~ms}]$ and $[t(i)+75 \mathrm{~ms}, t(i)+250 \mathrm{~ms}]$ [28]. This is the maximum-length segment without QRS complex, considering a maximum HR of $200 \mathrm{bpm}$ [28].

The component representing the fECG was then automatically selected by maximizing the $\mathrm{SNR}_{\text {out }}$ while minimizing the difference between the estimated HR and the expected value, i.e., $150 \mathrm{bpm}$. The ground truth of the R-peak locations was detected by applying the same algorithm on the simulated fECG signals before mixing them with $\mathrm{mECG}$ and noise. The detection error is then calculated by

$$
\text { Detection error }=\frac{\mathrm{FP}+\mathrm{FN}}{\mathrm{N}_{\text {peaks }}}
$$

where FP denotes false positive indicating wrongly detected peaks, FN denotes the false negative indicating missing peaks, and $\mathrm{N}_{\text {peaks }}$ is the total number of peaks.

Different levels of system noise, $\mathrm{SNR}_{\text {in }}$ from -12 to $2 \mathrm{~dB}$ in steps of $2 \mathrm{~dB}$, were simulated to examine the influence of system noise on the performance of the whole measurement chain. For each noise level, a varying number of capacitive electrodes, from 8 (the first two columns in Fig. 4) to 20 in steps of 4 (one adjacent column), were also simulated. The effects of the number of channels on the performance of the proposed ICA and R-peak detection algorithms were then investigated. In order to have only one independent variable, the MAs did not change for different $\mathrm{SNR}_{\mathrm{in}}$ and the number of channels. For each condition, the simulator was independently tested ten times, and the average $\mathrm{SNR}_{\text {out }}$ and detection error were considered. 
a

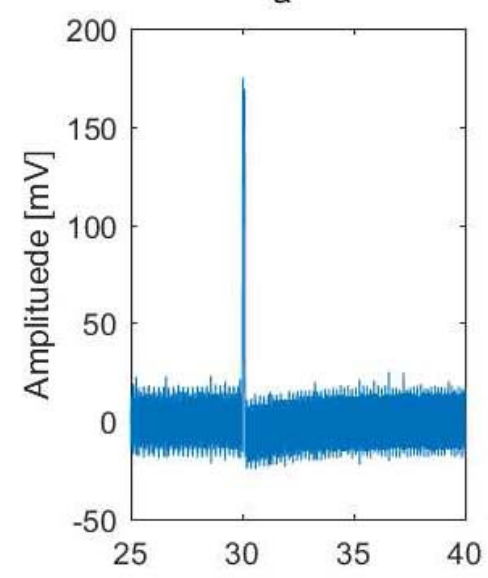

e

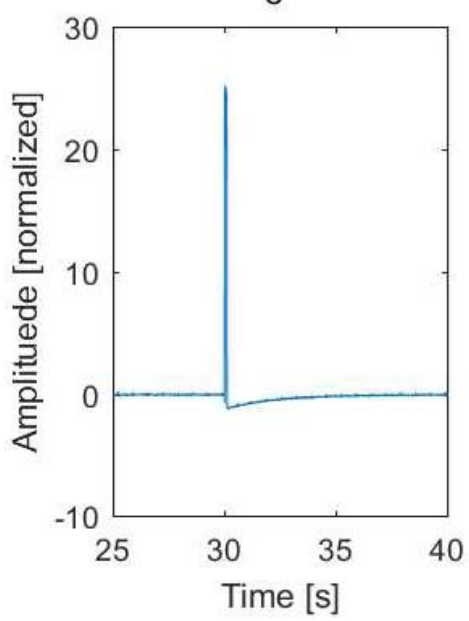

b

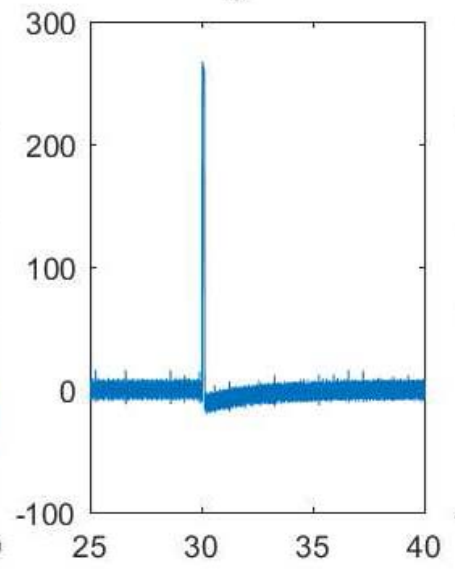

f

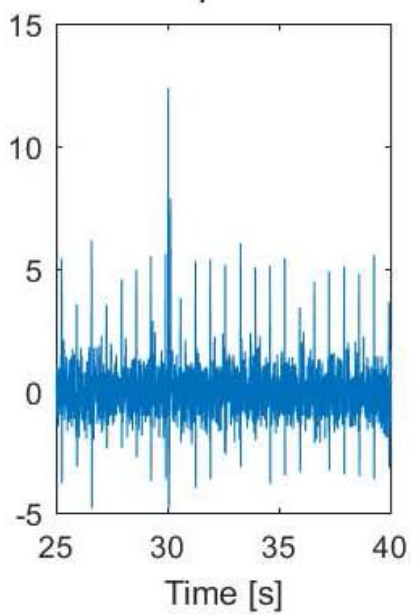

C

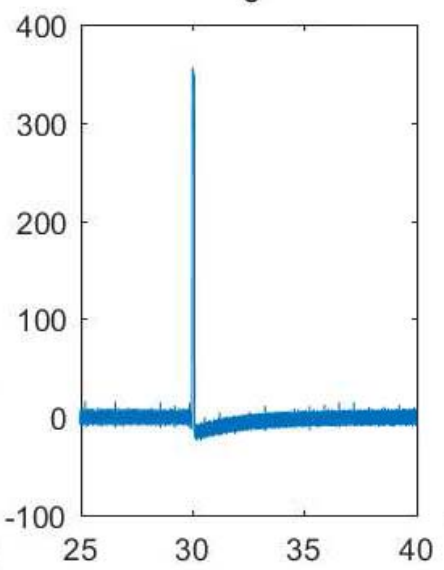

g

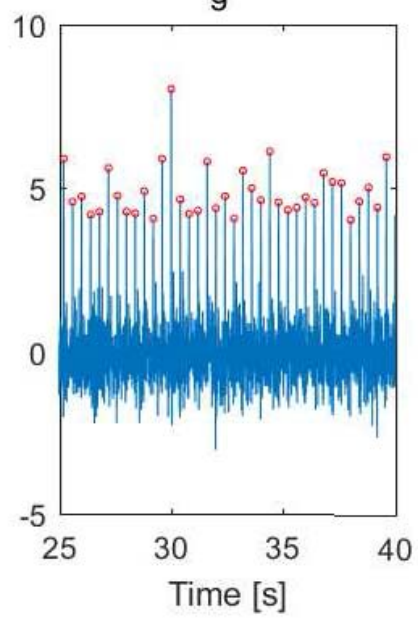

d

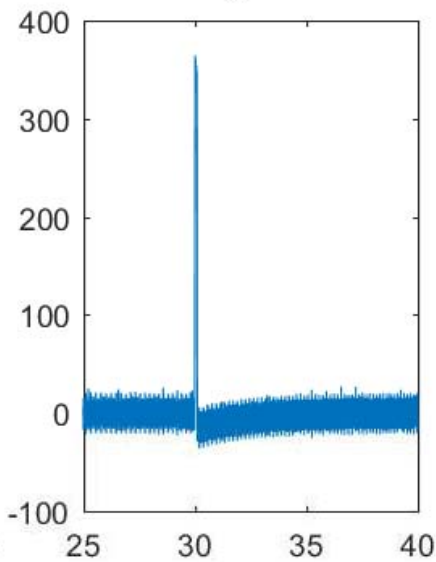

Fig. 7. Simulated signals (a)-(d) before and (e)-(h) after ICA. The signal amplitude after ICA is normalized with respect to its standard deviation. Eight channel recordings are used as the input of the ICA, and, for better visibility, only four input channels and four ICA components are shown here.

\section{RESULTS}

Fig. 5 shows a mixture of maternal and fetal ECG appearing on the surface of the volume conductor (Fig. 4). The peakto-peak amplitude of the fECG is around $5 \mu \mathrm{V}$, in line with previous studies [29]. The time-varying HRs for the mother and the fetus introduced by (9) are shown in Fig. 6. The maternal HR varies over time around an average value of $90 \mathrm{bpm}$ while the fetal HR varies around $150 \mathrm{bpm}$.

Fig. 7(a)-(d) shows four examples of the simulated signals before applying ICA. Each signal contains the fetal and maternal ECG, MAs due to longitudinal sensor movement modeled by a chirp motion and static charge modeled by a short current pulse, system noise modeled by white Gaussian noise, and the quantization noise introduced by the ADC. The fetal and maternal ECG signals are not visible due to their small amplitudes as compared to the simulated MAs. Fig. 7(e)-(h) shows four outputs of ICA, in which two components representing the fetal and maternal ECG signals can be identified, as shown in Fig. 7(g) and (f), respectively. Furthermore, the R-peaks in the fECG component are clearly visible and can be detected by the low complexity R-peak detection algorithm [28].
The influence of the number of channels and hardwareintroduced system noise, $\mathrm{SNR}_{\mathrm{in}}$, on ICA performance and $\mathrm{R}$-peak detection has also been investigated. For a fixed level of system noise, increase in the number of channels leads to increased SNR of the extracted fECG, $\mathrm{SNR}_{\text {out }}$, and, therefore, better R-peak detection, as shown in Fig. 8. In addition, for a fixed number of channels, higher $\mathrm{SNR}_{\text {out }}$ and better R-peak detection are observed with decreased system noise (increased $\mathrm{SNR}_{\text {in }}$ ), as shown in Fig. 9. The overall results showing the influence of different system-noise and number of channels on the SNR of the extracted fECG is given in Fig. 10, while the influence of different system noise and the number of channels on R-peak detection error is shown in Fig. 11.

\section{Discussion}

A multichannel simulator modeling the full fECG measurement chain using capacitive sensors has been realized in this article. The main factors that may affect fECG measurements, such as the position of the fetus, the number and position of the detecting electrodes, MAs, and the noise of the recording hardware [30], have been modeled and can be independently adjusted in the realized simulator. The realized simulator provides a suitable platform to develop dedicated 


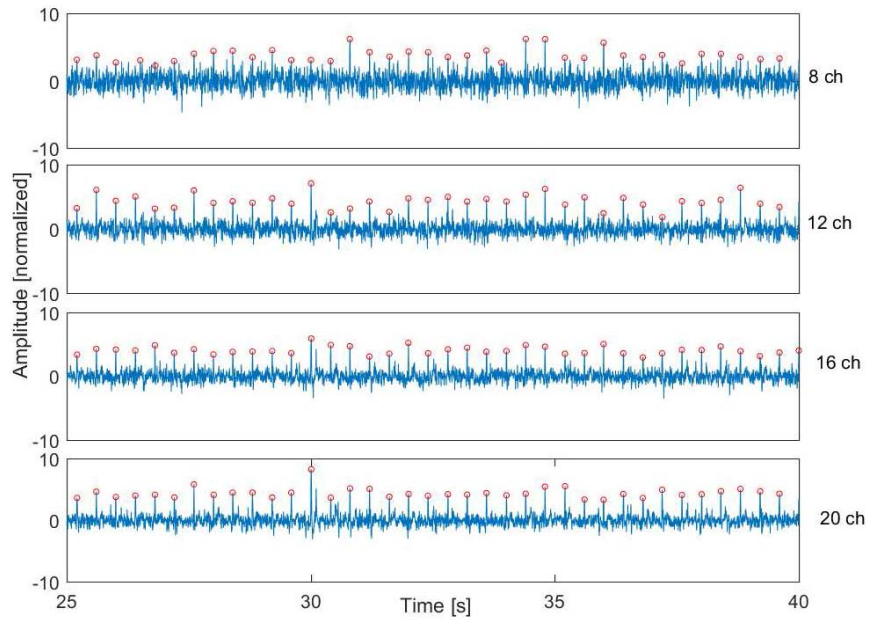

Fig. 8. R-peak detection on the extracted fECG with varying number of channels but fixed MAs and system noise $\mathrm{SNR}_{\mathrm{in}}-10 \mathrm{~dB}$. The signal amplitude is normalized with respect to its standard deviation.

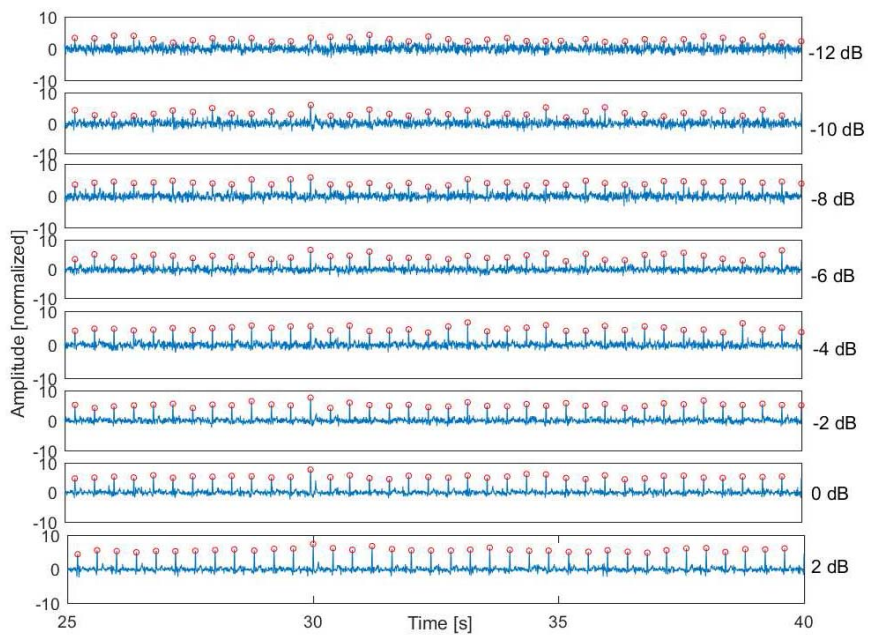

Fig. 9. R-peak detection on the extracted fECG with varying system noise, $\mathrm{SNR}_{\text {in }}$ from -12 to $2 \mathrm{~dB}$, but fixed MAs and number of channels at 12 . The signal amplitude is normalized with respect to its standard deviation.

algorithms for MA reduction and fECG extraction in capacitive measurements.

In fact, capacitive electrodes have been extensively suggested for adult ECG measurements [6]-[9]. However, fECG measurement systems using capacitive electrodes have been rarely reported in the literature. MAs pose the main challenges hampering the realization of a robust capacitive ECG measurement system [11]. The significantly smaller amplitude of the fECG further increases the complexity of capacitive fECG measurements. In one of our previous studies, an adaptive filtering method was proposed for MA reduction in single-lead capacitive ECG recorded on adults [17]. This article builds on the same capacitive sensing principle as proposed in [17]. However, considering the significantly smaller amplitude of the fECG, the single-lead capacitive sensor in [17] has been extended to a multichannel sensor matrix in this article in order to record the spatial information of the signals. This extension enables the possibility of applying BSS algorithms, i.e., ICA, to extract the fECG by exploring the spatial relation of the signals. Our results suggest that the ICA algorithm has

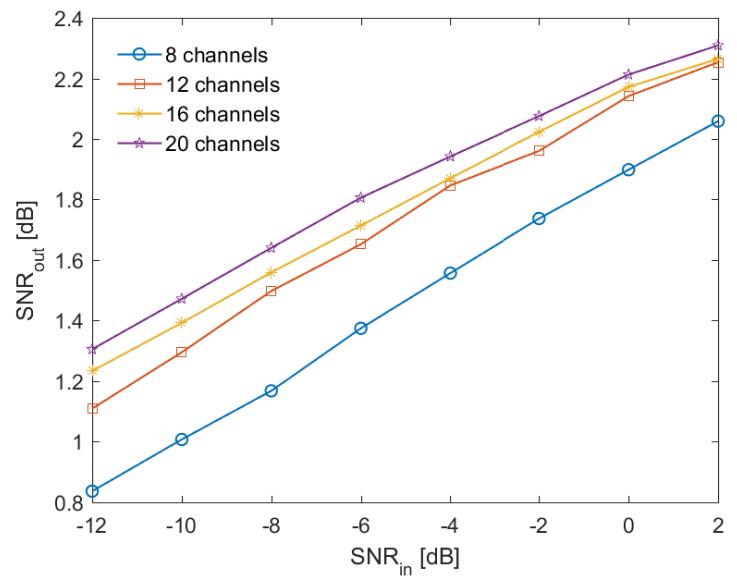

Fig. 10. SNR of the extracted fECG with varying system noise and number of channels but fixed MAs for each channel.

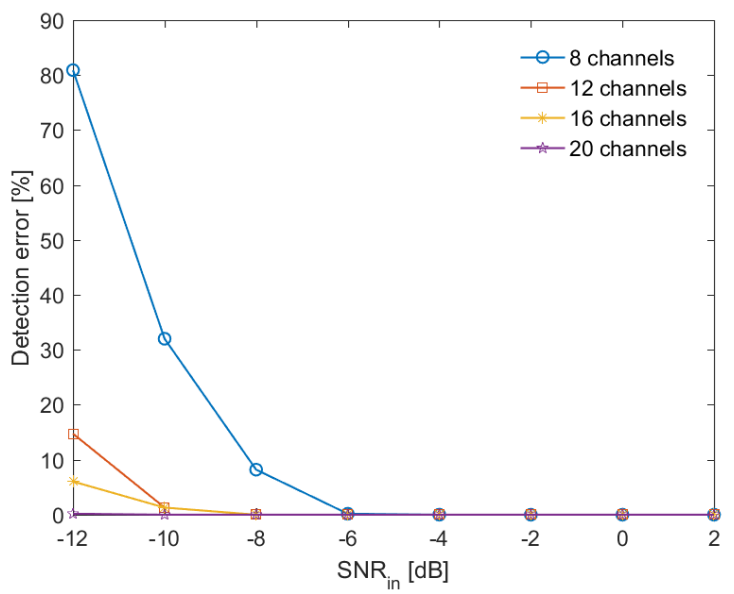

Fig. 11. Detection errors for varying system noise and number of channels but fixed MAs for each channel.

the potential to extract a fECG signal such that accurate fHR detection is feasible.

In general, the amplitude of an fECG signal is very small, i.e., in the order of few $\mu \mathrm{V}$ [29]. Consequently, fECG measurements require a recording system with very low system noise. In our simulator, white Gaussian noise is introduced to mimic the system noise of the recording hardware. The effects of different levels of system noise on the performance of fECG extraction and R-peak detection has also been investigated. Our results suggest lower system noise produce higher $\mathrm{SNR}_{\text {out }}$ in the extracted fECG and, therefore, lower R-peak detection error. Furthermore, our results show that, by using ICA, it is possible to extract the fECG from multichannel capacitive recordings with a negative $\mathrm{SNR}_{\text {in }}$, relaxing noise requirements for hardware design.

In theory, the number of sources should be less than the number of channels in order for an ICA algorithm to work properly. Considering the number of sources, i.e., six, simulated in this article, six channels are sufficient for reconstructing different sources. Given the electrode configuration in the simulator, $4 \times 5$ matrix (Fig. 4), a minimum of eight channels (two columns) was adopted in order to take a square matrix. Moreover, our results indicate that a larger number of electrodes lead to better fECG extraction and R-peak detection. 
However, increasing the number of recording electrodes may increase the complexity and power consumption of the recording hardware, which may become unsuitable for ambulatory monitoring. As a consequence, a tradeoff between the fetal HR detection accuracy and hardware complexity should be taken into account in different applications.

Furthermore, it should be noticed that the maternal and fetal ECG signals on the body surface are the projection of the VCG waveforms through the volume conductor (10), which depends mainly on the relative position between the detecting electrode and the VCG sources. As a result, the position of the recording electrodes can influence the quality of the whole capacitive measurement system [30]. Therefore, the effects of electrode position should be addressed in future studies. Moreover, the projection matrix in (10) is assumed to be uniform, and the variation in the permittivity of the volume conductor due, for instance, to the presence of the vernix caseosa has not been considered. This is the main limitation of the adopted ECG model and should also be addressed in future studies.

In the present simulator, two main MAs originating from longitudinal and lateral movement of the electrodes are considered. A chirp motion ranging between 0.2 and $10 \mathrm{~Hz}$ is adapted to mimic the longitudinal movement by assuming movement of the human body to be limited below $10 \mathrm{~Hz}$ [27]. In the meantime, the effects of the lateral movement of the electrodes, e.g., the triboelectricity, are modeled by a charge transfer between the body-electrode interface, as suggested in [12]. However, MAs in real measurements can be more complicated and cannot be fully modeled in our simulator. In vivo validation remains needed in future studies for a complete evaluation of MA effects.

In addition to MAs, power-line interference has also been considered in the multichannel simulator. The amplitudes of both MAs and power-line interference adopted in the simulator are in the dynamic range of the recording hardware, avoiding saturation in the amplifier. Therefore, most of the power-line interference can be removed by subtracting the reference (channel 21) from each active channel. However, the subtraction does not reduce differential-mode interference. Furthermore, large power-line interference and MAs may present in extreme measurement circumstances, which may saturate the front-end amplifier. Since no signal processing algorithm can correct the effects of amplifier saturation, the dynamic range of the front-end amplifier should be carefully considered during hardware design in order to prevent saturation. Furthermore, other noise sources, such as the electromyographic noise, from the mother should also be considered in future studies. Moreover, the dc offset of the amplifier and its time and temperature drift are not considered in the simulator since their frequency is very slow and can, therefore, be easily filtered out in the digital domain.

It is important to notice that the realized multichannel simulator in this article comprises of existing individual models, such as the ECG model, the capacitive electrode model, the FastICA, and the R-peak detection algorithm. Each of these models has been analyzed and evaluated by previous works [11], [14], [20], [25], [28]. The main novelty of this article is to integrate these well-established models in order to realize a full fECG measurement chain by multichannel capacitive sensors. Based on the realized simulator, we demonstrate, for the first time, the feasibility of extracting the fECG signals from noisy capacitive measurements using ICA. The main focus of this article is the full fECG measurement chain rather than the individual model. In fact, dedicated algorithms for mECG cancellation and fECG enhancement after ICA are available in the literature [31], [32]. By integrating those algorithms into our simulator, it is reasonable to expect further improvement in the fECG extraction and R-peak detection.

\section{CONClusion}

A dedicated simulator modeling the full fECG measurement chain by capacitive sensors is realized in this article. A BSS algorithm is proposed for capacitive fECG extraction from a noisy measurement, including both MAs and system noise. Our results predict good fECG extraction and R-peak detection by the proposed BSS algorithm. In addition, lower system noise and higher number of channels lead to better fECG extraction and R-peak detection. A maximum increase of $0.5 \mathrm{~dB}$ in the SNR and decrease of $80.7 \%$ in the R-peak detection error are observed with increased electrode number from 8 to 20. Our results provide useful insights for the hardware design of a capacitive fECG measurement system. Dedicated algorithms for fECG enhancement are available and can be easily integrated into the simulator, possibly leading to improved fECG extraction and R-peak detection. Finally, in vivo evaluation is required before introducing a capacitive system for real applications.

\section{REFERENCES}

[1] E. Malacova et al., "Risk of stillbirth, preterm delivery, and fetal growth restriction following exposure in a previous birth: Systematic review and meta-analysis," BJOG, Int. J. Obstetrics Gynaecol., vol. 125, no. 2, pp. 183-192, 2017.

[2] M. J. Stout and A. G. Cahill, "Electronic fetal monitoring: Past, present, and future," Clinics Perinatol., vol. 38, no. 1, pp. 127-142, 2010.

[3] A. Lopez and P. C. Richardson, "Capacitive electrocardiographic and bioelectric electrodes," IEEE Trans. Biomed. Eng., vol. BME-16, no. 1, p. 99, Jan. 1969.

[4] L. Leicht, B. Eilebrecht, S. Weyer, S. Leonhardt, and D. Teichmann, "Closed-loop control of humidification for artifact reduction in capacitive ECG measurements," IEEE Trans. Biomed. Circuits Syst., vol. 11, no. 2, pp. 300-313, Apr. 2017.

[5] J. Kirchner, S. Pfeiffer, and G. Fischer, "Passive capacitive ECG sensing: Assessment of signal quality during different types of body movement," in Proc. IEEE Int. Symp. Med. Meas. Appl. (MeMeA), Jun. 2018, pp. $1-5$.

[6] X. Yu et al., "A multi-modal sensor for a bed-integrated unobtrusive vital signs sensing array," IEEE Trans. Biomed. Circuits Syst., vol. 13, no. 3, pp. 529-539, Jun. 2019.

[7] I. D. Castro, R. Morariu, T. Torfs, C. Van Hoof, and R. Puers, "Robust wireless capacitive ECG system with adaptive signal quality and motion artifact reduction," in Proc. IEEE Int. Symp. Med. Meas. Appl. (MeMeA), May 2016, pp. 1-6.

[8] T. Wartzek, B. Eilebrecht, J. Lem, H.-J. Lindner, S. Leonhardt, and M. Walter, "ECG on the road: Robust and unobtrusive estimation of heart rate," IEEE Trans. Biomed. Eng., vol. 58, no. 11, pp. 3112-3120, Nov. 2011.

[9] J. Werth, A. Serteyn, P. Andriessen, R. M. Aarts, and X. Long, "Automated preterm infant sleep staging using capacitive electrocardiography," Physiolog. Meas., vol. 40, no. 5, Jun. 2019, Art. no. 055003. 
[10] Y. M. Chi, T.-P. Jung, and G. Cauwenberghs, "Dry-contact and noncontact biopotential electrodes: Methodological review," IEEE Rev. Biomed. Eng., vol. 3, pp. 106-119, Oct. 2010.

[11] J. Ottenbacher and S. Heuer, "Motion artefacts in capacitively coupled ECG electrodes," in Proc. World Congr. Med. Phys. Biomed. Eng., Munich, Germany, Sep. 2009, pp. 1059-1062.

[12] T. Wartzek, T. Lammersen, B. Eilebrecht, M. Walter, and S. Leonhardt, "Triboelectricity in capacitive biopotential measurements," IEEE Trans. Biomed. Eng., vol. 58, no. 5, pp. 1268-1277, May 2011.

[13] A. Serteyn, R. Vullings, M. Meftah, and J. W. M. Bergmans, "Motion artifacts in capacitive ECG measurements: Reducing the combined effect of DC voltages and capacitance changes using an injection signal," IEEE Trans. Biomed. Eng., vol. 62, no. 1, pp. 264-273, Jan. 2015.

[14] J. Behar, F. Andreotti, S. Zaunseder, Q. Li, J. Oster, and G. Clifford, "An ECG simulator for generating maternal-foetal activity mixtures on abdominal ECG recordings," Physiolog. Meas., vol. 35, no. 8, p. 1537, 2014.

[15] R. Sameni, G. D. Clifford, C. Jutten, and M. B. Shamsollahi, "Multichannel ECG and noise modeling: Application to maternal and fetal ECG signals," EURASIP J. Adv. Signal Process., vol. 2007, no. 1, pp. 043407-1-043407-14, Dec. 2007.

[16] L. Xu et al., "Adaptive motion-artifact reduction in capacitive ECG measurements by using the power-line interference," in Proc. IEEE Int. Symp. Med. Meas. Appl. (MeMeA), Jun. 2018, pp. 1-5.

[17] L. Xu, C. Rabotti, Y. Zhang, S. Ouzounov, P. J. A. Harpe, and M. Mischi, "Motion-artifact reduction in capacitive heart-rate measurements by adaptive filtering," IEEE Trans. Instrum. Meas., vol. 68, no. 10, pp. 4085-4093, Oct. 2019.

[18] G. Peng and M. F. Bocko, "Non-contact ECG employing signal compensation," in Proc. IEEE Biomed. Circuits Syst. Conf. (BioCAS), Oct./Nov. 2013, pp. 57-60.

[19] R. Vullings, C. H. L. Peters, M. J. M. Hermans, P. F. F. Wijn, S. G. Oei, and J. W. M. Bergmans, "A robust physiology-based source separation method for QRS detection in low amplitude fetal ECG recordings," Physiol. Meas., vol. 31, no. 7, pp. 935-951, 2010.

[20] P. E. McSharry, G. D. Clifford, L. Tarassenko, and L. A. Smith, "A dynamical model for generating synthetic electrocardiogram signals," IEEE Trans. Biomed. Eng., vol. 50, no. 3, pp. 289-294, Mar. 2003.

[21] M. Stridh and L. Sörnmo, "Spatiotemporal QRST cancellation techniques for analysis of atrial fibrillation," IEEE Trans. Biomed. Eng., vol. 48, no. 1, pp. 105-111, Jan. 2001.

[22] J. F. Cardoso and B. H. Laheld, "Equivariant adaptive source separation," IEEE Trans. Signal Procces., vol. 44, no. 12, pp. 3017-3030, Dec. 1996.

[23] J. Karhunen, E. Oja, L. Wang, R. Vigário, and J. Joutsensalo, "A class of neural networks for independent component analysis," IEEE Trans. Neural Netw., vol. 8, no. 3, pp. 486-504, May 1997.

[24] P. Comon, "Independent component analysis, A new concept?" Signal Process., vol. 36, pp. 287-314, Apr. 1994

[25] A. Hyvärinen, "Fast and robust fixed-point algorithms for independent component analysis," IEEE Trans. Neural Netw., vol. 10, no. 3 , pp. 626-634, May 1999.

[26] R. Bousseljot, D. Kreiseler, and A. Schnabel, "Nutzung der EKGSignaldatenbank CARDIODAT der PTB über das Internet," Biomedizinische Technik/Biomed. Eng., vol. 40, no. 1, pp. 317-318, 1995.

[27] H. W. Tam and J. G. Webster, "Minimizing electrode motion artifact by skin abrasion," IEEE Trans. Biomed. Eng., vol. BME-24, no. 2, pp. 134-139, Mar. 1977.

[28] M. J. Rooijakkers, C. Rabotti, S. G. Oei, and M. Mischi, "Lowcomplexity R-peak detection for ambulatory fetal monitoring," Physiol. Meas., vol. 33, no. 7, p. 1135, 2012.

[29] S. Song et al., "A low-voltage chopper-stabilized amplifier for fetal ECG monitoring with a 1.41 power efficiency factor," IEEE Trans. Biomed. Circuits Syst., vol. 9, no. 2, pp. 237-247, Apr. 2015.

[30] M. Rooijakkers et al., "Influence of electrode placement on signal quality for ambulatory pregnancy monitoring," Comput. Math. Methods Med., vol. 2014, Feb. 2014, Art. no. 960980

[31] S. M. M. Martens, M. Mischi, S. G. Oei, and J. W. M. Bergmans, "An improved adaptive power line interference canceller for electrocardiography," IEEE Trans. Biomed. Eng., vol. 53, no. 11, pp. 2220-2231, Nov. 2006.

[32] M. Varanini, G. Tartarisco, L. Billeci, A. Macerata, G. Pioggia, and R. Balocchi, "An efficient unsupervised fetal QRS complex detection from abdominal maternal ECG," Physiol. Meas., vol. 35, no. 8, pp. 1607-1619, 2014
Lin Xu (M'16) received the M.S. degree in electrical engineering from the University of Electronic Science and Technology of China, Chengdu, China, in 2010, and the Ph.D. degree from the Eindhoven University of Technology, Eindhoven, The Netherlands, in 2016.

$\mathrm{He}$ is currently an Assistant Professor with the School of Information Science and Technology, ShanghaiTech University, Shanghai, China. His current research interests include biomedical signal processing, with special emphasis on electromyography, neuromuscular conditioning, and ambulatory monitoring.

Chiara Rabotti (M'10) received the M.Sc. degree in electrical engineering from the University of Florence, Florence, Italy, in 2004, and the Ph.D. degree in the characterization of uterine activity during pregnancy from the Eindhoven University of Technology, Eindhoven, The Netherlands, in 2010.

She is currently a Senior Scientist with the Philips Research Laboratories, Eindhoven. Her current research interests include biomedical signal processing and modeling with an emphasis on electrohysterography, electromyography, and electrocardiography.

Yijing Zhang received the B.Sc. degree from Zhejiang University, Hangzhou, China, in 2015, and the M.Sc. degree from the Eindhoven University of Technology, Eindhoven, The Netherlands, in 2016, where she is currently pursuing the Ph.D. degree.

She is currently involved in biomedical front-end circuits for advanced ambulatory pregnancy monitoring.

Rik Vullings (M'10) received the M.Sc. degree (cum laude) in applied physics and the $\mathrm{Ph} . \mathrm{D}$. degree in electrical engineering from the Eindhoven University of Technology, Eindhoven, The Netherlands, in 2005 and 2010, respectively.

From 2010 to 2012, he was a Post-Doctoral Researcher with the Department of Electrical Engineering, Eindhoven University of Technology, where he has been an Assistant Professor since 2013. In 2010, he co-founded Nemo Healthcare, Veldhoven, The Netherlands, where he is currently leading the Signal Processing Team. His current research interests include signal processing for unobtrusive personal health solutions, with main applications in the fields of perinatology, cardiology, and somnology.

Mohammed Meftah is currently a Senior Scientist and the Project Lead with Philips Research Laboratories, Eindhoven, The Netherlands. He has a broad knowledge of biomedical sensors and systems, including unobtrusive sensing technologies, such as vital signs camera and capacitive electrophysiological sensors. He executed several clinical trials in different care settings, including unobtrusive monitoring of neonates in the NICU. His work has resulted in many publications and patents.

Sotir Ouzounov was born in Pazardzhik, Bulgaria, in 1973. He received the M.Sc. degree in electronics from the Sofia University of Technology, Sofia, Bulgaria, in 1997, and the Ph.D. degree in microelectronics from the Eindhoven University of Technology, Eindhoven, The Netherlands, in 2005.

Since 2008, he has been with the Philips Research Laboratories, Microsystems and Devices Group, Eindhoven. His current research interests include integrated, high-precision analog, and mixed-signal circuits and systems for electrophysiological measurements, medical imaging, and emerging biomedical applications.

Pieter J. A. Harpe (SM'15) received the M.Sc. and Ph.D. degrees from the Eindhoven University of Technology, Eindhoven, The Netherlands, in 2004 and 2010, respectively.

In 2008, he started as a Researcher with the Holst Centre, Imec, Eindhoven. Since 2008, he has been involved in ultralow-power wireless transceivers, with a focus on analog-to-digital converter research and design. In 2011, he joined the Eindhoven University of Technology, where he is currently an Associate Professor in low-power mixed-signal circuits.

Dr. Harpe is Co-Organizer of the yearly workshop on Advances in Analog Circuit Design and the Analog Subcommittee Chair of the ESSCIRC conference. He also served as an ISSCC ITPC member and an IEEE SSCS Distinguished Lecturer. He was a recipient of the ISSCC 2015 Distinguished Technical Paper Award.

Massimo Mischi (SM'10) received the M.Sc. degree in electrical engineering from La Sapienza University, Rome, Italy, in 1999, and the Ph.D. degree from the Eindhoven University of Technology, Eindhoven, The Netherlands, in 2004.

In 2007, he became an Assistant Professor with the Electrical Engineering Department, Eindhoven University of Technology, where he has been a Full Professor since 2018. He also chairs the Biomedical Diagnostics Research Laboratory, Eindhoven University of Technology. His current research interests include model-based quantitative analysis of biomedical signals.

In 2011, he was a recipient of a Starting Grant from the European Research Council for his research on angiogenesis imaging. He is the Chairman of the IEEE EMBS Benelux Chapter. 